\title{
Retour de Bournemouth : quelques réflexions historiennes sur The Connected Past: The Fu- ture of Past Networks? (22-25 août 2017)
}

\author{
Cécile Armand \\ Stanford University, Department of History \\ Submitted on August 27 $7^{\text {th }}, 2017$, published on March 23th, 2018
}

Actualités - News

Back from Bournemouth: A few historian reflections on The Connected Past: The Future of Past Networks? (22-25 August 2017)

Acteur, espace, histoire, numérique, réseau, temps.

Actor, Digital, History, Network, Space, Time.

Ce n'est pas toujours la détresse qui jette les bouteilles à la mer. Ici bien au contraire, c'est un élan d'enthousiasme et de gratitude qui pousse ce billet d'une rive à l'autre de la Manche. Un vent nouveau a soufflé sur la petite station balnéaire de Bournemouth, qui accueillait cette année la 7e édition de la conférence The Connected Past : The Future of Past Networks? (22-25 août 2017). Puissent l'énergie débordante des organisateurs et organisatrices, l'ingéniosité des participant.e.s et la richesse des projets présentés insuffler ce billet qui tente d'en rendre compte. D'où part cette bouteille ? Quel message porte-t-elle et quelle en est la portée ? Quelques précisions s'imposent d'abord sur le lieu d'où parle l'auteure et ses intentions.

Cet article ne traduit que le point de vue d'une jeune historienne récemment venue à l'analyse de réseaux, qui se tient encore au seuil de la 
pratique et s'interroge sur les applications possibles à son propre terrain de recherche - l'histoire de la publicité à Shanghai (1905-1949). Débutante en ce domaine, je ne m'aventurerai donc pas dans la technicité de ces outils. Sans être spécialiste des réseaux, je suis néanmoins engagée depuis plusieurs années dans ce qu'il est convenu d'appeler les « humanités numériques » : création d'un laboratoire junior " Nhumérisme » dédié à ces questions à l'ENS Lyon entre 2012-2015, animation d'un " atelier informatique et numérique pour historiens » à l'EHESS en 2014-2015, mobilisation d'une vaste palette d'outils dans le cadre de ma thèse (Armand, 2017) - des systèmes d'information géographique aux statistiques, en passant par les chronologies interactives ou la cartographie heuristique. Même si ma pratique relève davantage du bricolage que de l'expertise ou de la virtuosité, j'adopte ici le point de vue d'une historienne " numériste » qui observe depuis le rivage, avec une certaine fascination, la variété des usages et la créativité qu'autorisent ces outils de réseaux, comme l'ont exprimé les intervenant.e.s présent.e.s à cette conférence. Dans cette double perspective, la richesse des projets présentés à Bournemouth cette année apparaît à la fois comme un signe de la vitalité de ce champ spécifique (mais peut-on parler d'un champ au singulier face à une telle diversité de terrains, de pratiques et d'approches ?) et le révélateur de tendances plus globales, propres à l'environnement de recherche numérique qui est le nôtre aujourd'hui. A ce titre, trois aspects me frappent particulièrement.

Tout d'abord, le souci constant d'articuler les acteurs, le temps et l'espace. Au-delà des catégories qui structuraient très intelligemment le programme de la conférence (Ego Networks and Individuals, Accounting for Temporal Change, Networks and Geographical Space), cet enjeu affleurait en effet dans toutes les interventions, à des degrés et selon des modalités variables. Acteurs, temps, espace : telles sont les trois dimensions essentielles aux sciences sociales, qui restent néanmoins séparées dans la pratique. Héritage des partages disciplinaires, peut-être : au sociologue reviendrait l'étude des acteurs, tandis que l'espace appartiendrait au géographe et le temps à l'historien.ne. S'il confine à la caricature, ce partage simplifié des tâches n'en reste pas moins avéré, et généralement accepté comme allant de soi. Ce cloisonnement a jusqu'ici été entretenu par les 
outils et les pratiques numériques. On reproche souvent aux systèmes d'information géographiques classiques de produire des cartes statiques et désincarnées (Bodenhamer et al., 2010, 2015), aux outils de réseaux de manquer de dynamisme et de spatialité (Barthelemy, 2011 ; Bintliff, 2004 ; Haggerty, 2011 ; Oliver, 2016 ; Stark \& Vedres 2006 ; Yang \& Shekhar 2017), tandis que les chronologies "traditionnelles", qui restent aux marges des pratiques numériques, restent majoritairement linéaires, dépourvues d'une vraie dimension sociale et spatiale (Armand, 2014, 2015). Face à ces lacunes, tou.te.s les participant.e.s au Connected Past 2017 ont manifesté un effort réel pour joindre les trois angles de cette triade majeure: acteurs-espace-temps. Plusieurs projets entreprennent spécifiquement de mieux intégrer les deux premières dimensions, en articulant habilement les outils de réseaux et de cartographie : Cécile Rivals retrace ainsi l'évolution du parcellaire à Saint-Antonin-Noble-Val dans le Tarn et Garonne entre les XIVe et XIXe siècles ; Mark Groenhuijzen reconstitue les réseaux de transport locaux afin de préciser le tracé du limes romain ; Esther Lewis révise la place de Bristol dans l'histoire de l'hérésie, et repense l'opposition entre hérésie/orthodoxie à partir d'une étude fine des paroisses et de leurs interactions socio-spatiales sur le terrain. Au-delà, tou.te.s les participant.e.s ont exprimé leur souci de réinjecter une dimension dynamique dans les réseaux (Katherine Crawford propose par exemple d'utiliser les outils de réseaux pour scander les rythmes des processions rituelles à Ostie et observer leur inscription dynamique dans l'espace urbain), voire de réviser les périodisations établies à partir de l'analyse diachronique des interactions sociales en Grèce à l'époque classique (Denis Hazker). À cet égard, le site The Vistorian (Benjamin Bach) offre l'une des plateformes les plus abouties pour intégrer ces trois dimensions, en proposant des ressources directement accessibles à l'utilisateur ou l'utilisatrice. Par une interface très user-friendly, il est possible de librement charger ses données et les visualiser sous des formes diverses, afin d'observer leurs déploiements dans l'espace ou leurs recompositions dans le temps. Toutefois, ce projet est limité par l'absence d'un espace de stockage sur le site même, ce qui expose le chercheur ou la chercheuse au risque de perdre ses données et réduit également les possibilités de partage et de collaboration en ligne. Malgré leurs limites, toutes ces expérimentations n'en 
sont pas moins prometteuses et participent d'une tendance plus générale à l'intégration croissante de l'environnement numérique.

Cette conférence reflète ensuite les nouvelles compétences dont savent ou doivent désormais faire preuve les jeunes chercheurs et chercheuses à l'ère numérique. Les participant.e.s ont manifesté trois qualités en particulier, qui pourraient devenir de nouveaux standards académiques dans un avenir proche :

- Une créativité débridée, qui n'a pour seule limite que l'imagination du chercheur ou de la chercheuse. Comme l'a très justement rappelé Tom Brughmans au début de la conférence, l'analyste de réseau doit savoir quitter ses œillères et rester ouvert à toutes les applications possibles. Par exemple, un acteur n'est pas forcément un individu, mais peut être aussi une institution (publique ou privée), un objet matériel (marchandise) ou culturel (œuvre d'art ou de l'esprit). Comme pour tout outil numérique, les réseaux font appel à la créativité du chercheur. Ils l'invitent à s'approprier ces outils de manière créative pour les détourner à ses propres fins et leur inventer des usages inattendus voire inédits. C'est cet infini des possibles qui constitue le moteur même de la recherche.

- La nécessité d'expérimenter, qui s'exprime éminemment dans l'opération de modélisation - une question bien développée par Iza Romanowska et les deux physiciens Tim Evans et Ray Rivers. Toute modélisation implique la formulation, la mise à l'épreuve et la validation (ou non) d'hypothèses préalables. Cette procédure expérimentale était explicite dans tous les projets présentés. À l'ère numérique, les sciences sociales tendent ainsi de plus en plus vers les sciences expérimentales, et développent une démarche scientifique très proche de celle que décrivait l'historien Bernard Lepetit à propos de l'histoire quantitative à la fin des années 1980 (Lepetit, 1989).

- Des formes inédites d'interdisciplinarité, particulièrement visibles à travers les profils hybrides des intervenant.e.s, leurs formations atypiques et leur aptitude à collaborer avec des disciplines inhabituelles, du point de vue d'une historienne française 
qui a reçu une formation "classique»: physique, géologie, sciences neuronales, systèmes complexes (complexity science), informatique ou data-visualisation.

Enfin, un dernier étonnement vient ouvrir plus qu'il ne clôt ce retour critique : où sont les historien.ne.s ? Ce qui me frappe le plus au final, c'est le retrait sinon l'absence des historien.ne.s modernistes et contemporanéistes dans ce Connected Past qui les concerne pourtant au plus haut point. Pressentie en parcourant simplement la liste des intervenant.e.s, cette observation s'est confirmée en rencontrant physiquement les participant.e.s présent.e.s. Alors que les archéologues étaient majoritaires, les historien.ne.s se comptaient sur le doigt de la main et ne franchissaient qu'exceptionnellement la barrière médiévale (Esther Lewis). Comment comprendre ce déséquilibre ? Par les origines même de l'association Connected Past, fondée par un groupe d'archéologues pionniers en 2011, tandis que les historien.ne.s auraient leurs propres réseaux et se retrouveraient à d'autres occasions (Res-hist, GDR Analyse de réseaux en SHS, Historical Network Research) ? Par la permanence d'une coupure ancienne entre archéologues et historien.ne.s (non antiquisants), et au-delà, le maintien des frontières entre les quatre périodes conventionnelles de l'historiographie ? Faut-il y voir la persistance d'une conception très littéraire de l'histoire, et une certaine frilosité qui rend les historien.ne.s moins enclin.e.s à dialoguer avec l'archéologie, les sciences «dures » et l'informatique ? Est-ce parce qu'elles et ils ne mobilisent pas les mêmes sources (moins hétérogènes et moins dispersées que les archéologues) et raisonnent à des échelles différentes (les archéologues embrassant de plus longues durées et de plus vastes espaces) que leurs pratiques autour des réseaux peinent à se rencontrer ? Les historien.ne.s seraient-ils débordés par l'abondance des sources textuelles ? Auraientils mal digéré la parenthèse « cliométrique » des années 1960-1970 ? Plus généralement, est-ce en raison de la place centrale qu'y occupent l'expérimentation et des disciplines peu familières aux historien.ne.s que l'environnement numérique reste opaque à la majorité d'entre eux, quand il n'est pas tout simplement ignoré ou jugé incompatible avec le métier, tel qu'il est encore massivement pensé et pratiqué dans les milieux universitaires français? Cette ultime interrogation livre un bel objet de recherche 
à la sociologie des sciences et l'analyse de réseaux. Peut-on montrer l'existence de communautés autonomes, sinon indépendantes, dans ce champ hétérogène qu'est l'analyse de réseaux, et au-delà celui des pratiques numériques ? Où passent exactement les lignes de fracture et de jointure ? Comment expliquer les affiliations et les séparations intra et extra disciplinaires?

En conclusion, The Connected Past 2017 ouvre de nouveaux horizons à l'historienne consciente d'exercer dans un milieu technique en perpétuelle évolution, soucieuse de renouveler ses pratiques et curieuse de mettre l'analyse de réseau à l'épreuve du passé. Bien que bref et intense, le workshop des 22-23 août offrait une excellente mise en jambe, tant aux profanes solidement guidé.e.s dans leurs premiers pas avec Visone ou Netlogo, qu'aux praticien.ne.s chevronné.e.s qui pouvaient travailler à partir de leurs propres données. On ne peut que remercier les organisateurs et organisatrices d'avoir donné à une historienne - un peu métèque en ces terres numériques - l'opportunité de mettre les mains à la pâte, de réfléchir à la pertinence de ces outils pour ses propres recherches et d'être ainsi accueillie par une communauté réellement interdisciplinaire, dans un contexte de discussion aussi stimulant que chaleureux. Pour paraphraser le titre de cette édition 2017 («The Future of Past Networks »), The Connected Past semble bien promis à un bel avenir...

\section{Références}

Armand, C. (2017). "Placing the history of advertising »: une histoire spatiale de la publicité à Shanghai (1905-1949). Thèse de doctorat, ENS de Lyon.

Armand, C. (2015). Les chronologies numériques entre héritages et innovations : quelques implications méthodologiques et épistémologiques. Histoire et Informatique - Geschichte und Informatik, 18/19, 265-84.

Armand, C. (2014). Les représentations graphiques de la périodisation et les outils numériques: réflexions à partir d'un essai de périodisation de l'histoire publicitaire chinoise (1840-1949). In Gibert, S., Jean Le Bihan, J., \& Mazel, F. Revue Atala. Découper le temps : Actualité de la périodisation en histoire, 17, p. 249-262. 
Barthelemy, M. (2014). Discussion: Social and Spatial Networks . Les Nouvelles de l'archéologie, 135, 51-61.

Bintliff, J. (2004). Time, structure, and agency: the Annales, emergent complexity, and archaeology. In A companion to archaeology. Oxford: Blackwell (pp. 174-194).

Bodenhamer, David J., John Corrigan, J., \& Trevor M Harris, T.M. (2015). Deep Maps and Spatial Narratives. Bloomington: Indiana University Press.

Bodenhamer, David J., John Corrigan, J., \& Trevor M Harris, T.M. (2010). The Spatial Humanities: GIS and the Future of Humanities Scholarship. Bloomington: Indiana University Press.

Haggerty, J., \& Haggerty, S. (2011). Temporal Social Network Analysis for Historians - A Case Study. IMAGAPP/IVAPP, 207-17.

Lepetit, B. (1989). L'histoire quantitative : deux ou trois choses que je sais d'elle. Histoire $\mathcal{E}$ Mesure, 4/ 3, 191-199.

Oliver, D. (2016). Spatial network data: concepts and techniques for summarization. Switzerland: Springer.

Stark D., \& Vedres, B. (2006). Social Times of Network Spaces: Network Sequences and Foreign Investment in Hungary. American Journal of Sociology, 111/5, pp. 1367-1411.

Yang, K.S., \& Shekhar, S. (2017). Spatial Network Big Databases Queries and Storage Methods. Cham: Springer.

\section{Ressources multimédia}

Tous les liens ont été consultés le 27 août 2017.

Atelier informatique et numérique pour historien. Séminaire de l'EHESS en 2015-2016. http:/ / atelier-histnum.blogspot.fr/.

Bournemouth 2017 | Connected Past. http://connectedpast.net/otherevents/bournemouth-2017/.

GDR Analyse de réseaux en SHS. https://arshs.hypotheses.org/.

Historical Network Research. http://historicalnetworkresearch.org/welcome/.

MADspace | MADS. http://madspace.org/.

Netlogo. https://ccl.northwestern.edu/netlogo/.

Numérisme. Laboratoire junior dédié aux humanités numériques à l'ENS Lyon. http://dhlyon.hypotheses.org/ 
Pattern and Process I Spatial Simulation: Exploring Pattern and Process. http://patternandprocess.org/.

Réseaux et Histoire. http: / / reshist.hypotheses.org/.

The Vistorian. https://connectoscope.azurewebsites.net/vistorian/.

Visone. http://www.visone.info/. 